\title{
Developmental venous anomalies presented as chronic paroxysmal headache: a case report and brief review
}

\author{
Zheng Han ${ }^{1 a}$, Guozhong $\mathrm{Hu}^{2}$,Hongji $\operatorname{Tian}^{1}$,Songyan $\mathrm{Liu}^{1}$, Xuemei Han, ${ }^{\text {b }}$ \\ Zhongwen $L v^{3 c *}$
}

1.Departtment of Neurology, China-Japan Union hospital of Jilin University Changchun, Jilin, 130031, P.R.China

2.Departtment of pharmacy, China-Japan Union hospital of Jilin University Changchun, Jilin, 130031, P.R.China

3.Departtment of Radiology, China-Japan Union hospital of Jilin University Changchun, Jilin, 130031, P.R.China
a..12566825@qq.com
b.xuemei71267@sina.com
c.877505206@qq.com

*corresponding auther: Zhongwen Lv(877505206@qq.com)

Key words: Developmental venous anomalies,SWI,MRI, headache

Abstract cerebral venous malformation (CVM) is a rare disease which often result in misdiagnosis and missed diagnosis for its non-specific symptoms. we encountered a case of cerebral venous malformation with recurrent manifestations of perennial headache in 40-year-old woman.Through studying the main image features of Developmental venous anomalies (DVAs), We can improve the diagnostic level of CVM and strengthen the differentiation of headache,

\section{INTRODUCTION}

Since Developmental venous anomalies (DVAs) was first described by Pfannenstiel in 1887, scholars have done a lot of research about it. Patients who got this disease often present with non-specific symptoms such as headaches dizziness, etc ,which are no focal signs of localization, Even about $60 \%$ of patients has no obvious clinical manifestations. When a patient presents with the symptom of chronic recurrent headaches, clinicians tend to ignore screening of the cause, which often result in misdiagnosis and missed diagnosis .Here we report a case of DVAs with recurrent manifestations of perennial headache, and review of the relevant image literature, to improve the image interpretation and to trengthen the differential diagnosis of headache. 


\section{CASE REPORT}

The patient was a 40-year-old woman, who presented with one month severe headache ,dull reaction and low-grade fever. The headaches were described as severe and dull, mostly confined to the parietal-occipital area or to the right side of head with nausea, vomiting,, She also felt stiff neck and dull reaction at the same time. Before these symptoms ,she got a cold and suffered intermittent low-grade fever .The temperature in the afternoon did not exceed $38^{\circ} \mathrm{C}$,Her previous medical history included recurrent headaches for 20 years.which was diagnosed with "migraine" and the symtoms did not obviously be relieved after treatment, so the patient usually took oral painkillers to control symptoms.No positive signs were seen in the admission examination. Relevant auxiliary examination: cerebrospinal fluid: $230 \mathrm{~mm}$ water column pressure, leukocyte number 23 , the single core is greater than the multi-core, protein and sugar chloride is normal. Head CT (Fig.1) showed in the bilateral temporal Angle of lateral ventricle class round high density shadow, head MRI (Fig.2a, b, c) could be seen in the right lateral ventricle in the visible short T1 and long T2 and FLAIR hyperintensity, we could see on the right side of basal ganglia region long strips of low signal on T2WI and T2 FLAIR.According to the lumbar puncture and head CT and MRI results, we considered the headache worse a month ago was caused by meningeal stimulation from intraventricular hemorrhage and subsequent subarachnoid hemorrhage [1].In view of the ventricular hemorrhage of patient, and low T2WI signal shadow can be seen in vascular malformation, hemangioma, etc. [2]The patient was gaven the susceptibility weighted imaging( SWI) and MRA examination. Head SWI (Fig. 3a, b) can be seen many small expansion in veins group in the right basal ganglia, with a thick expansion of draining veins, and round low signal on the right after lateral ventricle Angle. MRA showed no abnormalities, From the above results, the diagnosis was cerebral venous malformation.

\section{DISCUSSION}

Common chronic secondary headaches can be seen in intracranial tumors, cerebral arteriovenous malformations and aneurysms, brain abscess, cervical headache and other diseases [3,4], in recent years due to a rare disease as previously thought of cerebral arteriovenous malformation which is ignored can also with chronic headache as the main performance. After studying a large number of domestic and foreign scholars, the disease has gradually been awareness. 
It is generally believed that cerebral venous malformation (CVM) is a histologically consist of vein and parenchymal completely, for local tissue functional venous drainage of the benign lesions, also known as brain developmental venous anomalies (DVA).Used to think that is a rare disease, according to wang ning, domestic study of CVM natural history and foreign major clinical reports, the disease is one of the vascular malformations in common, accounts for $7.3 \% \sim 63 \%$ of vascular malformations, less bleeding ratio [5]. Diverse clinical manifestations, mainly depends on the pathological changes, such as dizziness, headache, epilepsy, numbness. Most of the patients with no obvious clinical performance, are found. The patient with headache and intraventricular hemorrhage for clinical performance is relatively rare in the disease. The disease diagnosis is difficult in the past, but with the rapid development of imaging techniques and conventional MRI especially the wide application of functional magnetic resonance imaging (MRI), its diagnostic rate significantly increase.we need to observe the abnormalities of vascular bulky venous drainage, small expansion of medullary veins, presence of blood supply arteries, etc.on imaging, just for the diagnosis and differential diagnosis.

There is no specific performance of CVM on CT scan. Valavains summarizes the CT manifestations of the $55 \mathrm{CVM}$ cases and found that round high-density shadow more common, consider for the expansion of the pulp vein network [6]. CT is mainly used in the positioning and differential diagnosis. This case did not see the high-density expansion of medullary venous network in the CT, can be seen only after intraventricular hemorrhage hematoma formation.

The classic performance of CVM in MRI is multiple small medullary veins inflowing in one or more bulky drainage veins, but Yan Fengshan reported 16 cases of patients with MRI findings of CVM .According to the simple category, T1WI of MRI scan can show the typital features of CVM. For atypical CVM, MRI scan may has no specific performance, only showed low signal point ,so that it is easily missed [7]; Hu Congcong thought that most of the venous drainage in the TIWI and T2WI became low signal because of the blood vessel flow empty effect, It is not clear that part of high signal on T2WI or development .Some one considered the high signal is associated with the tiny venous drainage or artifact. Medullary veins showed, equal or low signal on T1WI, equal or high signal on T2WI, but this performance is low discovery rate in the clinic [8] .Wang Xiuling considered that: on T2-weighted sequences, medullary veins showed high signal, the large vein dilation showed low signal, the edge showed high signal, but only the larger CVM develop clear, 
CVM smaller and cortical region may develop unclear or does not develop [9]. Foreign literature also supports the MRI T1WI and T2WI of CVM were low signal.10] In our case there was not obviously exceptionally low signal on T1WI,but T2 - weighted images can clearly show long strips of low signal. In short, on the MRI scan most of the CVM may show abnormal signal, MRI diagnostic value of CVM higher than CT, but the diagnosis is of limited value. Magnetic Susceptibility Weighted Imaging (SWI) have greater value on trace intracranial hemorrhage, vascular malformations, hemangiomas and other central nervous system disorders[11], On SWI scan CVM can be clearly shown as a "jellyfish head". As we know,SWI has great value on the diagnosis of lots of central nervous diseases such as hemorrhage, intracranial vascular malformation and hemangioma [11], Scholars generally believe that CVM is a low flow vascular malformations, blood flow is slow, conventional MRI show high-flow vascular sensitivity, sensitivity to small low-flow vascular diameter decreased significantly. SWI based on MRI T2WI and joined the phase information, to enhance the sensitivity to magnetic material sensitivity, and the signal is not affected by the blood flow velocity; brain strongest magnetic susceptibility is mainly deoxyhemoglobin and hemosiderin, and intravenous Intravascular vascular malformations consists mainly of deoxy-hemoglobin blood, so SWI can easily show abnormal veins. SWI can manifest the vein of diameter less than $1 \mathrm{~mm}$ in the brain and is not affected by intravenous osteotomies [12]. In this case the SWI clearly show "jellyfish head", clearly show abnormal veins; basal ganglia also found multiple punctate low signal, suggesting that the patient has preexisting multiple old micro hemorrhage. Therefore, for CVM diagnosis SWI is significantly better than the conventional MR imaging techniques. With the Combination with conventional clinical MR ,SWI can improve the diagnosis rate of CVM.CVM performance in the DSA was found no abnormality in the arterial phase, in the vein of late show many small capillaries expansion of medullary veins, which import to significant expansion of central venous or sinus drainage as the so-called "jellyfish head "pattern, Located superficial cortical veins import sinus while deeper portion vein drain in the deep venous system of burglary subependymal [13]. DSA is generally considered the gold standard for the diagnosis of CVM, but failed due to the cavernous hemangioma in the DSA display $[14,15]$,therefore the merger cavernous hemangioma of CVM easily missed in DSA examination. 


\section{CONCLUSION}

when a patient has the focal symptoms such as headache, in addition to a detailed physical examination,we should think about possible disease and identify in detail,then give the suitable auxiliary examination, in order to avoid missed diagnosis and misdiagnosis. For CVM, general MRI can show abnormal signal, the auxiliary of SWI can give diagnosis, DSA is gold standard for diagnosis.But when the CVM merge other complex vascular malformation, MRI and SWI is the preferred imaging methods.

\section{References}

[1] Wujiang. neurology .Beijing: People's Health Press, 2005: 175-180

[2] Zhang Ying . Rare intracranial lesions in MRI signal characteristics study [J]. Journal of rare diseases, 2006, $13(5): 34$

[3]Nei yecheng. headache diagnosis [J] Japanese Medicine, 2007,28 (1): 16

[4] Zhang Xiang, Cheng Gang. common cause of secondary headaches and treatment [J] Chinese Journal of Internal Medicine, 2010,30 (6): 509

[5] Wang Ning. Cerebral venous malformation of natural history and treatment strategies [J]. Foreign Medical Sciences: Neurology, Neurosurgery volumes, 2002,29 (1): 35

[6] Wiklins RH et al.Neurosurgery Update II, NewYork, McGraw-Hill, 1991,134-139

[7]Yan fengshan,Li yongli,Zhang jiliang,et al.3.0 T MRI in the diagnosis of cerebral venous malformation value [J]. Journal of zhengzhou university (medical edition), 2009, 44 (4) : 891

[8] Hu congcong, Sun Jilin, Wu Jie,et al. Magnetic sensitive weighted imaging in the diagnosis of cerebral venous vascular malformation [J]. Journal of brain and neurological diseases, 2011, 12 (5) : 375

[9] Wang Xiuling, Zhang Zongjun, Zhu zongming,et al. Brain venous malformation CT and MRI diagnosis and evaluation [J] Journal of Medical Postgraduates, 2008,21 (9): 937

[10] Ragamonti D et al.J Neurosurg, 1988; 69 (4): 535-539

[11] Liu yaou, Yang yanhui .Magnetic sensitive weighted imaging in clinical application of the central nervous system [J]. Journal of medical imaging, 2007, (2) : 210

[12] Reichenbach JR, Venkatesan R, Schillinger DJ, et al.Small vessels in the human brain: MR venography with deoxyhemoglobin as an intrinsic contrast agent.Radiology, 1977,204: 272-277.

[13] Shen tianzhen.Neuroimaging,Shanghai: Shanghai Science and Technology Press, 2004: 
435-436

[14] Dai Jianping, Zhu mingwang. intracranial vascular malformations [J], Chinese Computed Medical Imaging, 2000, 6 (1): 44

[15] Garner TB, Curling OD Jr, Kelly DL Jr, et al.The natural history of intracrani al venous angiomas [J] .J Neurosurg, 1991,75: 715-722.
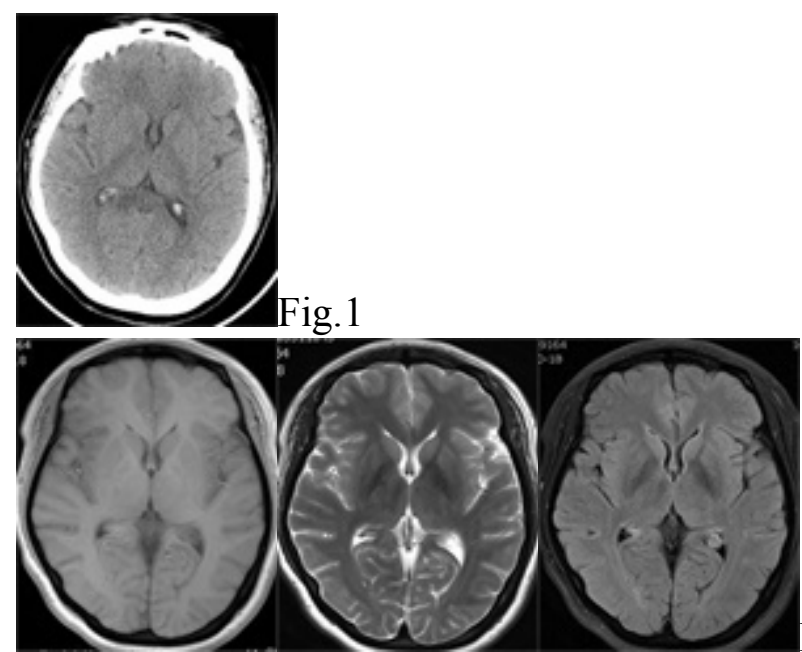

Fig.2a,b,c

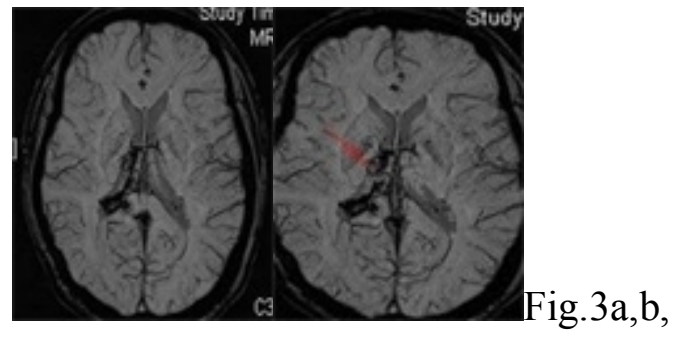

